Dos, paseos por los bosques narrativos (un lugar para la ficción)

\title{
De abismos y puentes: intimidad y memoria en Adrián Bravi y Maximiliano Mariotti
}

\author{
Palmieri, Massimo
}

Massimo Palmieri Sobre el autor Universidad Nacional de Córdoba, Argentina

El hilo de la fábula

Universidad Nacional del Litoral, Argentina

ISSN: $1667-7900$

ISSN-e: 2362-5651

Periodicidad: Anual

vol. 19 , núm. 21, 2021

revistaelhilodelafabula@fhuc.unl.edu.ar

Recepción: 28 Marzo 2021

Aprobación: 04 Julio 2021

DOI: https://doi.org/10.14409/hf.v0i21.10562
Resumen: Adrián Bravi y Maximiliano Mariotti, ambos en condición de sujetos migrantes, publican sendas obras narrativas en el idioma de adopción: Bravi, argentino, en italiano y Mariotti, italiano, en español. Ambos autores se colocan en una línea de tensión entre lenguas y culturas debido a su condición de sujetos migrantes crecidos y formados en un continuum semiótico distinto del que eligen para sus carreras de escritores. $\mathrm{Su}$ posición fronteriza los pone en una condición peculiar con respecto a la lengua que usan. La afirmación de Mariotti en uno de sus cuentos, «hay un abismo entre dar y recibir, y el abismo más angosto es el más difícil de salvar» (Mariotti, 1982:149), parece ser particularmente adecuada para describir esta relación entre dos lenguas de la misma familia romance cuya aparente cercanía contribuye a la construcción social de una otredad cargada de estereotipos. Cambio de lengua y literatura de la migración establecen especiales relaciones que dinamizan particulares tensiones dentro del canon pero también van deconstruyendo y re-construyendo mundos que se vinculan con la condición de migrante y refractan permanentemente la presencia del yo que escribe tratando de construir puentes para conectar memoria e identidad y contribuir a franquear abismos. En este artículo se tomarán algunos aspectos de las obras de los dos escritores que resultan particularmente significativas en este sentido.

Palabras clave: Literatura, Migración, Lengua, Identidad, Memoria.

Abstract: The purpose of this article is to analyze some works by two Italian-Argentine authors: Adrián Bravi and Maximiliano Mariotti, who publish narrative works in the adopted language: Bravi, Argentine, in Italian, and Mariotti, Italian, in Spanish. Both authors stand on a line of tension between languages and cultures due to their condition as migrant subjects grown up and formed in a semiotic continuum different from the one they choose for their writing careers. Their frontier position puts them in a peculiar condition towards the language they use. Mariotti's statement in one of his stories, «there is an abyss between giving and receiving, and the narrowest abyss is the most difficult to bridge» (Mariotti, 1982:149), seems to be particularly adequate to describe this relationship between two languages of the same romance family whose apparent closeness contributes to the social construction of an «otherness» full of stereotypes. The reading hypothesis is that change of language and literature of migration establish special relationships that dynamize particular tensions within the canon but also deconstruct and re-construct worlds that are linked to the condition 


\begin{abstract}
of migrant and permanently refract the presence of the self who writes trying to build bridges to connect memory and identity and contribute to bridge abysses. This article will take some aspects of the works of the two writers that are particularly significant in this sense.
\end{abstract}

Keywords: Literature, Migration, Language, Identity, Memory.

\section{Introducción}

El tema de este artículo pertenece al campo general de la literatura comparada y en especial a la literatura de migración que vincula de manera compleja el fenómeno del cambio de lengua en textos literarios producidos en italiano por el argentino Adrián Bravi y en castellano por el italiano Maximiliano Mariotti. El corpus está constituido por dos cuentos publicados en 10 de guerra (1982), de Maximiliano Mariotti y otros tantos relatos de Adrián Bravi publicados con el título Variazioni straniere (2015b) y traducidos al español en Córdoba como Después de la línea del Ecuador (2015a). Ambos autores se colocan en una línea de tensión entre lenguas y culturas debido a su condición de sujetos migrantes crecidos y formados en un continuum semiótico distinto del que eligen para producir textos con valor estético preponderante.

El fenómeno del cambio de lengua es un problema relevante de la literatura contemporánea. Nuestro referente es la obra de George Steiner, Extraterritorial, que vincula el fenómeno del cambio de lengua y la extraterritorialidad al paradigma de la tradición moderna. Los escritores extraterritoriales encuentran en el desplazamiento lingüístico características de experimentación e innovación. Es el caso de escritores como Nabokov, Beckett, Borges pero también de Italo Svevo, Ezra Pound, o Juan Rodolfo Wilcock.

Cambio de lengua y literatura de migración establecen relaciones que dinamizan particulares tensiones dentro del canon. Las nuevas voces que desde varios lugares del planeta se desplazan, afirmando paulatinamente nuevas instancias descolonizadoras y polifónicas desde una literatura transculturalizada que posibilita investigar a fines de reconstruir cómo construye al otro un escritor que cambia de lengua, cuáles son sus recorridos expresivos en una lengua que en un primer momento operó como límite para ingresar a la «cultura otra», hasta conseguir hacer de ella misma un instrumento de acercamiento y reafirmación.

A partir de varios interrogantes relativos al diálogo intercultural-intertextual en las obras literarias producidas por estos escritores migrantes, la hipótesis central es que la literatura producida por Mariotti y Bravi, sujetos migrantes que cambian de lengua, permite leer los modos en que se construye al «otro». La polifonía de voces que van constituyendo el imaginario colectivo está presente en modo privilegiado en textos elaborados por sujetos situados en un espacio fronterizo de la semiosfera (Lotman, 1996). Las huellas de esta condición de sujetos situados en la frontera semiótica, están presentes en las elecciones lingüísticas y literarias y permiten advertir los niveles de conflictividad inherentes a todo dialogismo. Adrián Bravi y Maximiliano Mariotti publican sendas obras narrativas en el idioma de adopción: Bravi, argentino, en italiano y Mariotti, italiano, en español. Su posición fronteriza los pone en una condición peculiar con respecto a la lengua que usan. 
La afirmación de Mariotti, que en uno de sus cuentos declara «hay un abismo entre dar y recibir, y el abismo más angosto es el más difícil de salvar» (Mariotti, 1982:149), parece particularmente adecuada para describir esta relación entre dos lenguas de la misma familia romance cuya aparente cercanía contribuye a la construcción social de una otredad cargada de estereotipos. Además de impulsar las tensiones dentro del canon literario, cambio de lengua y literatura de la migración van de-construyendo y re-construyendo mundos que se vinculan con la condición de migrante y refractan permanentemente la presencia del yo que escribe tratando de construir puentes para conectar memoria e identidad y contribuir a franquear abismos. En este artículo se tomarán algunos aspectos de las obras de los dos escritores que resultan particularmente significativas en este sentido.

\section{De abismos y puentes}

Los diez cuentos de Maximiliano Mariotti, publicados en 1982 por la editorial Emecé de Buenos Aires bajo el título general de 10 de guerra, se desarrollan todos en el contexto del segundo conflicto mundial, hecho histórico vivido en primera persona por el autor, que durante esa época pasaba sus años de la adolescencia en una Italia azotada por la crudeza de unos acontecimientos bélicos que involucraban también a gran parte de la población civil.

La frase que citamos en el apartado anterior, se refiere a uno de dichos cuentos, precisamente el sexto en orden cronológico, titulado «El tanque de Zaratustra» (Mariotti, 1982:135-149), cuyo eje diegético es representado por el cruce entre dos caracteres opuestos que corresponden a dos personajes que ocupan diferentes lugares jerárquicos dentro del ejército alemán: el soldado simple Kramer y el teniente Hopfel. Ambos personajes conforman, juntos al soldado Weiss, la tripulación de un tanque alemán en retirada del frente ruso durante los últimos años del conflicto. Pero mientras el primero de los dos representa a un hombre rudo y de baja cultura, de profesión verdulero, el segundo es un doctor en filosofía y entre ellos parece no haber ningún tipo de comunicación. Kramer, hombre concreto e ignorante, considera a Hopfel un cobarde pusilánime, por su conducta más propensa a la reflexión que a la acción. En el momento en que el tanque no puede seguir más su camino por problemas mecánicos, el teniente ordena a la tripulación de abandonar la máquina, pero Kramer no soporta esta actitud considerando el acto de abandonar el tanque en manos del enemigo una intolerable falta de coraje.

El teniente impone al soldado que obedezca y lo acusa de insubordinación, ordenando al otro miembro de la patrulla que lo controle bajo la amenaza de una pistola para que siga el camino. De repente Kramer se percata de que el «cobarde» teniente Hopfel se ha dirigido hasta el tanque para sacrificarse y hacerse volar junto a la máquina, para evitar que caiga en manos del enemigo. $\mathrm{He}$ aquí donde aparece la frase «hay un abismo entre dar y recibir, y el abismo más angosto es el más difícil de salvar. Así susurró Zaratustra en los oídos de Hopfel, llevado de la mano de Nietzsche, quien proclamaba que existía una moral entre los que mandaban y los que obedecían» (Mariotti, 1982:149).

Sin embargo, la moral no logra salvar el abismo entre los dos seres. Kramer nunca llegará a comprender el gesto de Hopfel y volverá a su vida habitual 
después del conflicto sin haber aprendido ninguna lección ética. Quizás porque la guerra nunca nos puede enseñar algo de verdad y con más razón las diferencias resultan insalvables, aunque parezcan estrechas en aquel horroroso precipicio del alma que es un conflicto bélico. Es justamente esta la frase que fundamenta uno de los ejes de nuestro análisis cuando hacemos referencia a los abismos lingüísticos y culturales que se abren a veces precisamente donde los bordes parecen más estrechos y en cambio crean puentes y salidas al cruzar el espejo de una uniformidad ilusoria para encontrar soluciones en las diferencias creativas.

En el relato que acabamos de sintetizar, el abismo parece definitivamente derrumbar todo intento de levantar un puente entre dos hombres, así como la guerra no permite el diálogo entre las partes en pos de un supuesto derecho a defenderse que muchas veces se confunde con el deber de atacar, sobre el cual se construyeron miles de conflictos generadores de muertes. Por otro lado, se perciben claramente, por parte de Mariotti, intentos de poner en comunicación dos mundos que aparecen demasiado distantes entre sí para comprenderse.

El más emblemático en este sentido es otro cuento perteneciente a la colección ya citada, titulado «Armand» (Mariotti, 1982:65-81), que narra esencialmente de una amistad imposible entre un muchacho italiano y un soldado alemán. Amistad imposible porque el soldado alemán era un enemigo, debía considerarse entonces el contrario de un amigo y, sin embargo, como observa el chico en un pasaje del relato «él era un hombre bueno, demasiado bueno para ser un suboficial alemán metido en una dura guerra» (Mariotti, 1982:67).

Los diálogos entre los dos personajes se revelan como intentos de poner en comunicación dos mundos destinados a quedar incomunicados, pero Armand, joven hombre de veintiséis años, elige a Sebastián, adolescente de diecisiete, como a un referente para manifestar la absurdidad de la guerra, desde su visión de ex profesor de botánica en Dortmund.

El soldado alemán se expresa en una lengua incierta pero claramente comprensible, que nosotros leemos como el español elegido por Mariotti para narrar la historia, aunque el idioma que el soldado hablaba con su joven amigo, en ese contexto, debiera ser el italiano. Con su habla huérfana de artículos y conjugaciones verbales, mas a través de palabras llenas de humana sabiduría, Armand afirma que «flores existen en todas partes: diferente forma y especie, pero iguales en función (...) Con hombres ser igual, especie y razas no idénticas, pero idéntica función vivir mundo para mejor conocerlo» (Mariotti, 1982:72-73)

A cierto punto del cuento, Armand advierte a Sebastián de que está prevista una razzia (sic) por parte del ejército alemán en represalia de una acción de la resistencia y le aconseja alejarse por unos días. Esa advertencia le salvará la vida al joven italiano quien, sin embargo, a la vuelta no encontrará más a su amigo alemán cuya única huella será un enorme cráter dejado por la bomba que había alcanzado de lleno su tanque. Las palabras que había usado el hombre para advertir el muchacho sobre el peligro que corría eran las siguientes: «Váyanse por el camino a Fibbialla. Ningún alemán se meterá por ese lado» (Mariotti, 1982:74). Sebastián, con otros jóvenes, se va para la aldea que le había aconsejado Armand, huyendo de las represalias de los militares teutónicos.

En esta parte del libro aparece claramente un aspecto autobiográfico. En primera instancia el chico del cuento es el único entre los personajes principales 
de todos los relatos que tiene la edad que tenía Mariotti en tiempo de guerra, en segundo lugar, pudimos identificar claramente la correspondencia entre lugares $\mathrm{y}$ hechos de la ficción y las vivencias del autor por medio de un diario personal perteneciente al autor que llegó a nuestras manos durante la investigación.

El personaje de Armand, con un idioma despojado de vínculos gramaticales, logra trasmitirnos más profundamente que cualquier profesor de lengua con todos sus subjuntivos en orden, el absurdo horror de la guerra. Sin embargo, el trabajo filológico y semiótico-cultural de nuestro autor es notable, considerando que se pone en una triple frontera semiosférica que se transforma en un juego de espejos: un alemán que trata de explicar su pensamiento a un chico italiano expresándose en idioma español. Nos parece relevante, más allá del sugestivo intento de balbuceo que expresa de forma lograda la impotencia del hombre frente a sus propias aberraciones, la presencia de juegos de palabras que requieren una doble lectura. Para indicar la trampa expresiva del lenguaje verbal, Armand usa el término «rañas-telas», que en español correcto sería claramente «telarañas». Sin embargo, en italiano se escribe «ragnatele». Es decir que para hacer hablar al soldado un italiano incierto y no ortodoxo utiliza la posición formal italiana de la palabra compuesta logrando un efecto doblemente confuso en español donde la posición canónica es al revés.

Con este recurso «de frontera» Mariotti logra un mayor efecto de confusión, sus elecciones lingüísticas requieren de un doble filtro para ser interpretadas y nos develan una riqueza intercultural que a primera vista queda casi escondida detrás de parecidos simplificadores.

\section{La lengua como hermenéutica del mundo}

De forma especular al caso de Mariotti tenemos el de Adrián Bravi, escritor bonaerense trasplantado en Recanati, que tiene a su vez una notable producción de cuentos y ensayos publicados en italiano. En este caso nos vamos a focalizar en dos publicaciones que han reunido algunos de estos, es decir Variazioni straniere y Después de la línea del Ecuador, ambas publicadas en el año 2015.

La primera recopilación consiste en nueve relatos publicados por EUM, editorial de la Universidad de Macerata, ateneo en el cual Bravi desempeña su actividad de bibliotecario y, según nos ha revelado el mismo autor, nace como idea de un obsequio navideño a los empleados de la institución. En su mayor parte, los cuentos que componen el libro ya habían sido publicados individualmente en varias revistas impresas o virtuales y algunos de ellos figuran también, traducidos al español, en la otra publicación a la que hiciéramos referencia anteriormente.

El denominador común de todos los relatos es la reflexión acerca de la migración entendida también en su faceta de diversidad, exclusión y marginalidad. Se trata de nueve breves historias que tienen como eje el ser extranjero, aunque sea en su propia tierra, como es caso del viejo jubilado protagonista de «Io, il badato» (Bravi, 2015a:17-31).

Este cuento forma parte también de la recopilación Después de la línea del Ecuador, publicada por la Sofía Cartonera, editorial de la Facultad de Filosofía y Letras de la Universidad Nacional de Córdoba, casi contemporáneamente a la edición italiana, ambas pues, al cuidado de una institución universitaria. En realidad, la edición «cordobesa» de estos relatos precede por unos meses a la 
publicación italiana, debido a que durante el año anterior Adrián Bravi nos concedió el permiso de utilizar algunas de sus obras breves para traducirlas al español y publicarlas en Argentina a través de la editorial que utiliza el material recolectado por los cartoneros para confeccionar la tapa de sus libros. Este último compendio está conformado por seis cuentos traducidos, cinco de los cuales aparecen también, en su versión italiana original, en la publicación de la universidad de Macerata.

En el caso de «Io, il badato», la versión española lleva el título «Yo, el asistido» (Bravi, 2015a:45-55) y cuenta la historia de un hombre anciano ya no autosuficiente que, como pasa muy a menudo en una sociedad italiana siempre más integrada por personas mayores, debe recurrir al cuidado de una mujer para la cual en Italia se usa la palabra badante, que en este caso ha sido traducida con el término «asistente».

La debilidad, la marginalidad de este hombre indefenso que la enfermedad ha convertido en un ser aislado e incapaz de comunicar, lo acerca a la figura del extranjero, del «diverso», del que no encaja en la normalidad. Su final triste y al mismo tiempo irónico revela cómo la única persona que intenta hasta lo último consolarlo y cuidarlo a pesar de su impotencia frente a una absurda muerte violenta es la extranjera inmigrada, su asistente ucraniana, cuyo rostro dulce y lloroso es lo último que el viejo moribundo ve.

Las palabras clave alrededor de las cuales el autor construye el eje del relato, son las que tienen como raíz el verbo badare o sea «cuidar», «asistir» y todo el abanico semántico que abarca este término. Tan es así que la traducción del título original «Io, il badato», necesita un ajuste que lleva en español a la siguiente expresión «Yo, el asistido». En este pasaje podemos percibir uno de los posibles recorridos fundamentales de nuestra labor investigativa: el sondeo analítico entre los vericuetos de dos sistemas lingüísticos pertenecientes a la misma familia romance que por su intuitiva cercanía a veces no pueden salvar los abismos más estrechos que hacen de la comunicación un diálogo entre sordos que, sin embargo, por medio de la experimentación literaria puede transformarse en energía creativa.

Ahora bien, Bravi, como hace de manera casi sistemática, recupera expresiones comunes que describen realidades sociales y psicológicas vinculadas a una semiosfera, como en este caso la palabra badante, y construye a su alrededor un cuento en el que afloran muchas de las posibles implicaciones socioculturales. La palabra badante se traduce al español con «asistente» porque es el término que logra acercarse más al significado denotado del original italiano, sin embargo, necesita de algunas explicaciones contextuales que en la edición argentina se aplican por medio de una extensa nota a pie de página.

Esto se hace necesario a raíz del sentido que la palabra en cuestión ha adquirido en el contexto social de Italia a partir de los últimos veinte años, en relación con el aumento de la expectativa de vida por un lado y de la inmigración por el otro, por lo que se van identificando estereotipos y frecuencias de uso que hasta las últimas décadas del siglo pasado no tenían comparación posible. He aquí que Bravi aprovecha la ocasión para acuñar un neologismo, badato, que si bien no existe en el diccionario, se puede interpretar a la luz de la situación y no crea ninguna ambigüedad, sino más bien logra desplazar el discurso hacia la mirada del diverso, del marginal, en este caso el anciano, que no casualmente, a pesar de 
su imposibilidad de expresarse articulando palabras, declara de forma evidente su simpatía hacia la otra persona instalada en la periferia cultural: la extranjera, su asistente ucraniana.

De este modo el universo semiótico de las «asistentes» (le badanti) adquiere una nueva dignidad, desafiando el estereotipo construido por el imaginario colectivo de la semiosfera cultural italiana que las identifica como mujeres, en su mayoría del este de Europa, inmigradas por necesidad, más bien ordinarias, rudas y torpes. Bravi, en efecto, transforma la robustez física, estereotípicamente vista como elemento áspero, en una materna suavidad que envuelve al protagonista en una ternura cómplice y afectuosa.

Este es solo un aspecto de la potencialidad innovadora y refrescante del lenguaje de Bravi con respecto a lengua italiana literaria, porque se vislumbran también algunos virtuosismos debidos a la incursión del castellano en la lengua huésped, siempre en punta de pie, de manera no invasiva, como es el caso de la expresión «e patatìn e patatàn» (Bravi, 2015b:18), que nunca antes habíamos encontrado en italiano pero que puede perfectamente encajar en el discurso, en el ritmo y en la fonética.

Otro de los cuentos que aquí analizamos es el primero que aparece en ambas publicaciones y además da el título a la recopilación completa de la edición argentina, es decir «Dopo la línea dell'equatore» (Bravi, 2015b: 7-16), traducido como «Después de la línea del Ecuador»(Bravi, 2015a:3-11). El relato se construye sobre la historia de Franco Jarkiewicz, hijo de padre polaco y madre italiana, que emigra desde Italia hacia América a los pocos meses de vida, junto a sus padres.

Los hechos ocurren poco después de terminada la segunda guerra mundial. El padre de Franco, un soldado polaco de quien la madre se enamora durante el conflicto, está obligado a dejar Italia y elige como destino la Argentina. En el transcurso del viaje, poco después de la línea del Ecuador, en el barco se agotan las reservas de agua y muchos niños no logran sobrevivir. La madre de Franco, que lo está amamantando, se siente culpable para no haber podido salvarlos a todos con su leche. Los niños muertos son arrojados del barco envueltos en una sábana. Más de cincuenta años después, durante la crisis del 2001 en la Argentina, Franco está nuevamente obligado a emigrar haciendo el viaje al revés. Se encuentra de vuelta en el país que lo vio nacer pero que ahora le es extraño y cuyo idioma no puede comprender. Después de haber intentado diversos trabajos sin éxito, decide volver a la Argentina. Obligado a viajar por barco debido a la falta de dinero, morirá víctima de un infarto en el medio del océano y será arrojado al mar envuelto en una sábana blanca, como aquellos niños que la madre no había logrado salvar tantos años antes.

Se trata evidentemente de un relato circular, en el cual el protagonista es conducido por el destino hacia un final ineluctable y en donde la alteridad está constantemente presente. Desde la figura del padre, extranjero y alcohólico, que Franco casi no podrá conocer, su retorno a los orígenes en un país que le es ajeno y lo trata como tal, hasta aquella tensión al reconocimiento que lo vincula con el grupo de los otros, de los que no habían logrado llegar a destino. Curiosamente la palabra destino, que en español tiene dos sentidos, indicando por un lado la meta de un viaje, y por otro la parte de la historia de cada uno sobre la cual no tenemos 
control, en italiano significa una cosa sola: el diseño que va más allá de la voluntad humana de acuerdo al cual nuestras vidas toman ciertos caminos y no otros.

En el primer párrafo del cuento en italiano leemos: «nessuno ha immaginato il destino che nascondeva la vita di Franco Jarkiewicz» (Bravi, 2015b:7), en donde destino indica claramente el hado, lo incalculable, lo inevitable de la vida. La traducción dice: «ninguno imaginó el destino que escondía la vida de Franco Jarkiewicz» (Bravi, 2015a:3). Se entiende claramente que se habla de algo ineludible, pero a su vez se podría jugar sobre el hecho que la vida de Franco cambió muchas veces de rumbo hasta llegar al espacio en donde destino como lugar de arribo y destino como predestinación se encuentran en una perfecta circularidad. Es probable que el juego de palabras detrás del cual el relato se construye haya sido una de las chispas creativas para armar la estructura del cuento, como si un sistema lingüístico pudiera servir de recurso para cubrir un vacío semántico del otro, o por lo menos enriquecerlo. Esta situación se repite más de una vez en la narrativa de Bravi.

El autor nos explicó, en una charla, como el relato en cuestión tuviese algo de biográfico y como este suceso se lo contaba una tía que lo había sufrido en primera persona y quien nunca había llorado al contarlo en español, a pesar de la evidente congoja que al rememorarlo le causaba. Una sola vez lloró y fue cuando lo contó en italiano, el idioma en que había vivido la experiencia (Bravi, 2017.25). Como decir que la lengua no es solo una forma de describir al mundo sino también y sobre todo una condición del espíritu. En otro fragmento del texto leemos directamente en la versión traducida:

En ese período, a nadie se le ocurría rechazar un barco cargado de inmigrantes; es más, el gobierno de Perón alentaba la inmigración; se consideraba un recurso; alemanes, hebreos, italianos, turcos, españoles, franceses, llegaban de todo el mundo. Atracaban en Buenos Aires y después se desparramaban por el país (Bravi, 2015a:5).

La referencia a la situación actual es evidente. Sin polémicas, con la liviandad que define su estilo y nos hace recordar, como ya observamos, al Calvino de las Lezioni americane, Bravi subraya lo cíclico de una historia que nos parece nueva y sin embargo no lo es, haciéndonos notar cómo la memoria colectiva tienda a ser selectiva y a corto plazo. Franco es la personificación del otro. Otro para los lugares, extranjero en el país en donde crece y en aquello en donde nació. Otro para las personas, destinado a morir solo y a ser arrojado al mar, espacio por excelencia de la no identidad, cronotopo del viaje, a menudo trágico. En esta historia reconocemos el signo como posición ideológica, más allá de un estilo nunca retórico y que conlleva aquella liviandad indicada por Italo Calvino como uno de las exigencias de la literatura del nuevo milenio, liviandad que no es superficialidad en tanto no se opone a la profundidad sino a cierta pesadez que impide a la creatividad levantar vuelo (Calvino, 1988:5-30).

En el relato de Bravi se advierte cómo la dignidad del otro va más allá de la tolerancia. Se convierte en aceptación de la diversidad hasta reconocerse como parte de ella. Reconocer que nosotros mismos somos otros de alguien más, significa también comprender que en nuestro mundo hay espacio para el otro como parte de nosotros mismos. Este mensaje queda particularmente marcado en el cuento que no casualmente da el título a la publicación argentina, pero se puede reconocer en todos los relatos como eje, en tanto búsqueda de un diálogo que representa hoy una urgencia planetaria frente al fenómeno de las migraciones 
masivas que llevan las culturas a encontrarse, confrontarse y enfrentarse cada día más.

La patria entonces estaría representada por ese lugar de la memoria donde se construye y reconstruye nuestro yo y por ende, nuestra vida. Como observa el mismo Bravi en su ensayo La gelosia delle lingue,

Passare da una lingua a un'altra significa porsi di fronte a un rischio. . Ogni esperienza che facciamo con la lingua, sia essa straniera o propria, presuppone una rinascita e un punto di non ritorno. Non parliamo questa o quella lingua ma siamo in questa o quella lingua. ${ }^{1}$ (Bravi, 2017:8)

La lengua no es solo expresión y comunicación sino construcción de nuestro propio ser. El cambio de lengua, más aún en un escritor, implica una reformulación de las coordenadas culturales e individuales que inevitablemente se construyen y re-construyen a través de las experiencias vividas.

La memoria stessa è una forma della lingua. Si ricorda sempre all'interno di questa, e mai allo stesso modo in due lingue diverse. Compiere un tale passaggio significa diventare una specie di palinsesto. La nostra vita viene in qualche modo riscritta, reinterpretata alla luce della nuova lingua. ${ }^{2}$ (Bravi, 2017:9)

Se trata de un proceso paulatino, que sin embargo representa la esencia misma del viaje hacia el otro y en descubrimiento de lo otro, tanto en el sentido metafórico de movimiento interior, como en el acto del desplazamiento físico, así que «la stessa migrazione andrebbe considerata sotto il profilo linguistico, appunto perché è lì che s'inscrive la propria identità e la propria memoria» ${ }^{3}$ (Bravi, 2017:9).

La nueva lengua también puede representar una defensa contra un pasado de sufrimiento, un intento de superar momentos traumáticos vinculados al idioma nativo en que se vivieron, como era el caso del cuento de la tía, o de exiliados políticos, desaparecidos, sobrevivientes de los campos de concentración. Así como la lengua materna es una cápsula en que se envuelve de alguna manera el yo en exilio para defender su ser, la nueva lengua también lo ayuda «a trovare la propria libertà» (Bravi, 2017:172).

\section{Conclusiones}

En los casos que hemos analizado corroboramos que el marco de libertad constituido por la condición de sujetos que escriben en lengua no materna se puede confirmar en muchas elecciones estilísticas, sintácticas y lexicales, aunque de formas diversas en los dos autores.

En efecto, la experimentación lingüística que consideramos podría ser favorecida por el cambio de lengua, está bien presente en nuestros autores a pesar de sus notables diferencias. En los dos casos hemos evidenciado no solo su capacidad de mirar la lengua desde afuera, pesando las palabras y mirándolas con lupa, como expresara Borges (1980:37), sino también la operación especular de utilizar el idioma «otro» para mirar desde un lugar neutro hechos y fenómenos pertenecientes a una semiosfera que responde a otros códigos.

Las elecciones lingüísticas y literarias de estos escritores refractan de modo bien reconocible, en varias ocasiones que hemos analizado, su cualidad de sujetos que 
se encuentran en un lugar periférico en el cual se articulan distintas semiosferas, que se reproducen a través de conflictos, pero también de reconocimientos.

La condición de sujetos migrantes ubicados entre dos semiosferas de referencia, coloca efectivamente a Mariotti y Bravi en una posición fronteriza entre culturas que no albergan conflictos evidentes, pero viven a menudo de estereotipos muchas veces cristalizados en modismos, palabras y actitudes peculiares, pero a su vez empobrecedoras. Tanto Bravi como Mariotti saben mantener esa «distancia de seguridad» con respecto a los estereotipos más comunes en el diálogo intercultural aunque en Mariotti este distanciamiento se realiza en una adhesión al canon para evitar caer en abismos demasiados estrechos para evitarlos, mientras que en Bravi encontramos una disposición casi de aceptación afectiva hacia el estereotipo, debido seguramente al diverso lugar de ubicación pero también a una posición más abierta a las contaminaciones, vinculada quizás con exigencias de mestizaje e instancias interculturales presentes de manera muy fuerte en la sociedad actual.

\section{Referencias}

Borges, Jorge Luis (1980). Siete noches. Editorial Meló.

Bravi, Adrián (2015a). Después de la línea del Ecuador. La Sofía cartonera.

Bravi, Adrián (2015b). Variazioni straniere. EUM edizioni.

Bravi, Adrián (2017). La gelosia delle lingue. EUM edizioni.

Calvino, Italo (1988). Lezioni americane. Garzanti.

Lotman, Iuri (1996). La semiosfera I. Semiótica de la cultura y del texto. (Trad. Desiderio Navarro). Cátedra.

Mariotti, Maximiliano (1982). 10 de guerra. Emecé Editores.

Steiner, George (2009). Extraterritorial: ensayos sobre la literatura y la revolución del lenguaje. (Trad. Edgardo Rosso). Adriana Hidalgo Editora.

\section{Notas}

1 Pasar de una lengua a otra significa ponerse frente a un riesgo. . . Toda experiencia que hacemos con la lengua, ya sea extranjera o propia, presupone un renacimiento y un punto de no retorno. No hablamos esta o aquella lengua, sino que somos en esta o aquella lengua. Las traducciones al español en las notas son mias.

2 La memoria misma es una forma de la lengua. Recordamos siempre al interior de esta, y nunca de la misma manera en dos lenguas distintas. Realizar un paso similar significa convertirse en una especie de palimpsesto. Nuestra vida de alguna forma se re-escribe, se reinterpreta a la luz de la nueva lengua.

3 La migración misma debería considerarse bajo el perfil lingüístico, justamente porque allí se asientan la identidad y la memoria personal.

\section{Sobre el autor}

Doctor en Ciencias del Lenguaje con mención en Literatura Comparada e Interculturalidad. Docente titular de las cátedras de lengua italiana IV, didáctica especial I y Literatura Italiana I y II en la Facultad de Lenguas de la UNC. 